\title{
Activity of temocillin and 15 other agents, including fosfomycin and colistin, against Enterobacteriaceae in Hong Kong
}

\author{
Margaret Ip ${ }^{1}$ - Christopher K. Lai ${ }^{2}$ • Kitty S. C. Fung ${ }^{3}$ K-Tak Wong ${ }^{1} \cdot$ Chendi Zhu $^{1}$ • \\ Sebastien Van de Velde ${ }^{4}$ Dominic N. Tsang ${ }^{2} \cdot$ Peter Hawkey $^{5}$
}

Received: 27 June 2017 / Accepted: 9 August 2017 /Published online: 25 August 2017

(C) The Author(s) 2017. This article is an open access publication

\begin{abstract}
Limited data are available on temocillin susceptibilities in Enterobacteriaceae from Asian countries where antimicrobial resistance is prevalent. The in vitro activities of temocillin and 15 commonly used antimicrobials against 613 non-duplicate blood $(n=310)$ and urine (with clinically significant bacteriuria; $n=303$ ) isolates of Enterobacteriaceae from patients who attended 3 out of 7 clusters of public hospitals of the Hospital Authority, Hong Kong, during 2015/2016 were tested. Minimum inhibitory concentrations (MICs) were determined by Clinical and Laboratory Standards Institute (CLSI) microbroth dilution (agar dilution with fosfomycin). For temocillin, MICs were also obtained using the British Society of Antimicrobial Chemotherapy (BSAC) microbroth dilution method and interpreted using the BSAC breakpoints. Overall, 93.0\% (570) isolates were susceptible to temocillin using BSAC systemic breakpoint $(\leq 8 \mathrm{mg} / \mathrm{L})$ and all except 2 isolates were susceptible using the urinary breakpoint $(\leq 32 \mathrm{mg} / \mathrm{L})$. The extended spectrum
\end{abstract}

Electronic supplementary material The online version of this article (https://doi.org/10.1007/s10096-017-3091-8) contains supplementary material, which is available to authorized users

Margaret Ip

margaretip@cuhk.edu.hk

1 Department of Microbiology, Chinese University of Hong Kong, Prince of Wales Hospital, Shatin, Hong Kong

2 Department of Pathology, Queen Elizabeth Hospital, Kowloon, Hong Kong

3 Department of Pathology, United Christian Hospital, Kwun Tong, Hong Kong

4 Eumedica S.A., Brussels, Belgium

5 Institute of Microbiology and Infection, University of Birmingham, Birmingham B15 2TT, UK beta-lactamase (ESBL) positivity rate was $23.2 \%$ (118 out of 508 E. coli, Klebsiella spp., Proteus spp.). Temocillin resistance rate to ESBL-positive isolates was $16.1 \%$ using the systemic breakpoint of $\leq 8 \mathrm{mg} / \mathrm{L}\left(\mathrm{MIC}_{50}\right.$ and $\mathrm{MIC}_{90}$ were $8 \mathrm{mg} / \mathrm{L}$ and $16 \mathrm{mg} / \mathrm{L}$ respectively). Two isolates $(1 \mathrm{E}$. coli, temocillin MIC $64 \mathrm{mg} / \mathrm{L}, 1$ Klebsiella sp., MIC $32 \mathrm{mg} / \mathrm{mL}$ ) were resistant to meropenem and possessed the NDM-5 and KPC-2 genes respectively. Other susceptibility rates were: amoxicillin/clavulanate $(59.1 \%)$, trimethoprim/sulfamethoxazole (62.5\%), ciprofloxacin (71.5\%), ceftriaxone $(75.4 \%)$, nitrofurantoin $(76.4 \%)$, gentamicin $(78.3 \%)$, cefepime $(81.1 \%)$, ceftazidime $(83.5 \%)$, piperacillin/tazobactam $(86 \%)$, colistin $(88.8 \%)$, tigecycline (89.4\%), fosfomycin (92.8\%), ertapenem (99.0\%), amikacin $(99.2 \%)$ and meropenem (99.7\%). Temocillin may be a useful alternative for the treatment of infections caused by ESBL and multi-drug-resistant Enterobacteriaceae in Hong Kong, particularly as resistance rates to ciprofloxacin, nitrofurantoin and piperacillin/tazobactam are high.

\section{Introduction}

The global pandemic of CTX-M extended spectrum betalactamases (ESBLs) has driven rates of multi-drug-resistant Gram-negative bacteria (MDRGNB) to unprecedented levels, particularly in Asia [1]. This is exemplified by recent data reporting an ESBL rate in E. coli of $66 \%$ in China [2], whereas in Hong Kong, $20 \%$ of E. coli and $15.4 \%$ of $K$. pneumoniae causing bacteraemia in hospitalized patients had been reported to be ESBL-positive isolates [3, 4]. The spread of carbapenemase-producing Enterobacteriaceae brought further concerns around carbapenem overuse together with the need for heightened infection control.

Temocillin has been introduced into the UK and a number of European countries for the treatment of infections caused by 
ESBL-producing Enterobacteriaceae [5, 6]. Temocillin, a narrow spectrum penicillin (6- $\alpha$-methoxy-ticarcillin) with intrinsic stability to AmpCs, ESBLs and some carbapenemases, has been considered potentially a "carbapenem-sparing agent", especially in the treatment of ESBL-producing enterobacterial infections of the urinary tract [7]. However, limited data exist on the activity of temocillin against Enterobacteriaceae in Asian countries and China. We thus sought to evaluate the in vitro activity of temocillin and commonly used antimicrobials (including fosfomycin, tigecycline and colistin) against clinical isolates of Enterobacteriaceae in patients with urinary tract infections and/or bacteraemia in Hong Kong hospitals. Most laboratories in this region perform antimicrobial susceptibilities based on the Clinical and Laboratory Standards Institute (CLSI) method, and as the susceptibility to temocillin had been based on the British Society of Antimicrobial Chemotherapy (BSAC)-defined MIC breakpoints for Enterobacteriaceae [8], we also examined and compared its activity using the microbroth dilution methods according to the CLSI and the BSAC methods.

\section{Materials and methods}

\section{Bacterial isolates}

Non-duplicate isolates of Enterobacteriaceae from blood $(n=310)$ or urine (with clinically significant bacteriuria; $n=303$ ) from patients who attended 3 out of 7 clusters of hospitals of the Hospital Authority in Hong Kong between January 2015 and January 2016 were examined. These clusters provide public hospital care with a catchment area for over $50 \%$ of the Hong Kong population, and isolates included in this study were from the Prince of Wales Hospital (PWH), North District Hospital (NDH), Shatin Hospital (SH), Queen Elizabeth Hospital (QEH) and United Christian Hospital (UCH). Bacterial identification was based on conventional biochemical methods and/or use of commercial systems, e.g. API, Vitek or MALDITOF, as established in each hospital's accredited microbiology laboratory. The isolates included were E. coli (349), Klebsiella spp. (109), Proteus spp. (50), Enterobacter spp. (35), Citrobacter spp. (25), Salmonella spp. (17), Morganella spp. (16), Serratia spp. (11), Providencia spp. (1).

\section{Antimicrobial susceptibility testing}

The minimum inhibitory concentrations (MICs) to 16 antibiotics, namely, temocillin, amoxicillin/clavulanate, trimethoprim/sulfamethoxazole, ciprofloxacin, nitrofurantoin, gentamicin, amikacin, ceftriaxone, ceftazidime, cefepime, piperacillin/tazobactam, colistin, tigecycline, fosfomycin, ertapenem and meropenem, were performed and interpreted according to the CLSI microbroth dilution method [9, 10]. For fosfomycin, Mueller-Hinton agar supplemented with glucose-6-phosphate was used for MIC determination according to the CLSI agar dilution method $[9,10]$. As there are currently no CLSI breakpoints for temocillin, the MICs to temocillin were performed using the BSAC's microbroth dilution method [11] and interpreted according to the BSAC systemic and urinary breakpoints ( $\leq 8 \mathrm{mg} / \mathrm{L}$ and $\leq 32 \mathrm{mg} / \mathrm{L}$ respectively) [8]. ESBL detection was confirmed by the combination disks diffusion method according to the CLSI, using cefotaxime/ceftazidime with and without clavulanate [10]. E. coli NCTC 10418 and $P$. aeruginosa NCTC 10662 strains were included as controls in the BSAC method [11]. MIC values of temocillin by the BSAC vs CLSI methods were compared and MIC agreement was defined as previously described [12].

\section{Results}

The antimicrobial susceptibilities of 613 isolates of Enterobacteriaceae are listed in Supplementary Tables 1 and 2. The susceptibility rates to temocillin according to the BSAC method were $93.0 \%$ (570 out of 613) and 100\% using the systemic $(\leq 8 \mathrm{mg} / \mathrm{L})$ and urinary $(\leq 32 \mathrm{mg} / \mathrm{L})$ breakpoints respectively. The ESBL positivity rate was $23.2 \%$ (118 out of $508 \mathrm{E}$. coli, Klebsiella spp., Proteus spp.). The temocillin $\mathrm{MIC}_{50}$ and $\mathrm{MIC}_{90}$ for these ESBL-positive isolates were $8 \mathrm{mg} / \mathrm{L}$ and $16 \mathrm{mg} / \mathrm{L}$ respectively. The temocillin resistance rate for ESBL-positive
Fig. 1 Distribution of temocillin minimum inhibitory concentration values of 613 bacterial isolates according to the British Society of Antimicrobial Chemotherapy (BSAC) and Clinical and Laboratory Standards Institute (CLSI) methods

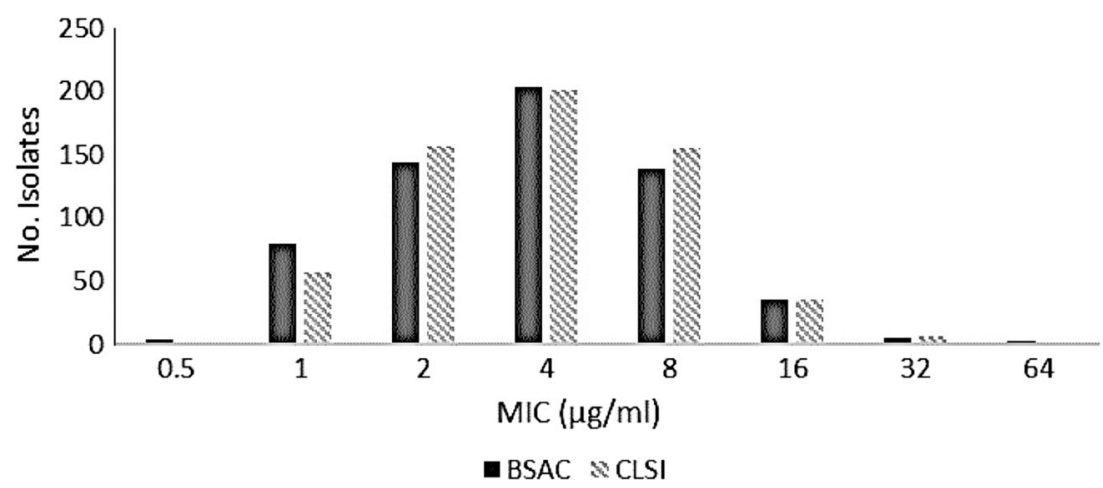


isolates was $16.1 \%$ using the systemic breakpoint of $\leq 8 \mathrm{mg} / \mathrm{L}$. Other antimicrobial susceptibility rates were: amoxicillin/ clavulanate $(59.1 \%)$, trimethoprim/sulfamethoxazole $(62.5 \%)$, ciprofloxacin $(71.5 \%)$, ceftriaxone $(75.4 \%)$, nitrofurantoin $(76.4 \%)$, gentamicin $(78.3 \%)$, cefepime $(81.1 \%)$, ceftazidime (83.5\%), piperacillin/tazobactam (86\%), colistin (88.8\%), tigecycline $(89.4 \%)$, fosfomycin $(92.8 \%)$, ertapenem $(99.0 \%)$, amikacin $(99.2 \%)$ and meropenem $(99.7 \%)$. Only two isolates (1 E. coli (TMO MIC $64 \mathrm{mg} / \mathrm{L}$ ), 1 Klebsiella species (temocillin MIC $32 \mathrm{mg} / \mathrm{mL}$ ) were resistant to meropenem and possessed the NDM-5 and KPC-2 genes respectively.

When MIC values of temocillin obtained by the CLSI and BSAC methods were compared, all MIC values obtained by the CLSI method fell within a two-fold dilution of the MIC values obtained using the BSAC method (Fig. 1). A good correlation, $\mathrm{R}^{2}=0.87$, was obtained between the two methods and the overall percentage susceptibilities based on the CLSI microbroth method remained unchanged.

\section{Discussion}

Temocillin provides a therapeutic option in the management of infections caused by multi-drug-resistant Enterobacteriaceae. In a previous study involving six hospitals in England, temocillin was used in the treatment of urinary tract and bloodstream infections caused by ESBL/AmpC-producing Enterobacteriaceae with high clinical and microbiological cure rates (of $91 \%$ and $92 \%$ respectively) [5]. Temocillin was given $2 \mathrm{~g}$ twice daily irrespective of the ESBL/AmpC production by the infecting organism(s), reaffirming its potential application as a carbapenem-sparing agent [5].

There is a paucity of susceptibility data for temocillin in the Asian Pacific region. A Korean study revealed a temocillin susceptibility rate of $96.8 \%$ (335 out of 346) in E. coli from community-acquired urinary tract infection (UTI) [13], whereas in another study solely focusing on ESBL-producing E. coli from patients with communityacquired acute pyelonephritis [14], susceptibilities varied with $100 \%$ susceptibility $(N=11)$ in CTX-M-14 strains, while only $72.7 \%$ ( $n=11)$ of CTX-M-15 strains were susceptible. In a recent study from Singapore, a high susceptibility rate of $95 \%$ for both E. coli and K. pneumoniae was reported when breakpoints for uncomplicated UTIs were applied; however, the rates were substantially lower when "systemic infection" breakpoints were used [15].

Currently, the resistance rates to agents such as ciprofloxacin, nitrofurantoin and piperacillin/tazobactam in Hong Kong are high, necessitating a switch to reserved agents such as colistin and fosfomycin for treating infections caused by MDRGNB. Temocillin may be a useful alternative for the treatment of infections caused by ESBL- and multi-drugresistant Enterobacteriaceae in Hong Kong, particularly as a "carbapenem-sparing agent". To our knowledge, this study is the first to report the use of the CLSI microbroth dilution method for the testing of temocillin and a good correlation of MIC values with temocillin between the BSAC and CLSI microbroth dilution methods was obtained.

Funding This study was supported by Belpharma S.A. Luxembourg (PI to MI).

\section{Compliance with ethical standards}

Competing interests $\mathrm{PMH}$ has received speaker and meeting attendance support from Eumedica. MI has received funds from Pfizer, Eumedica and MSD for support of studies, presentations at conferences and/or consultancy.

Ethical approval Approval of this study was obtained from the Joint Chinese University of Hong Kong - New Territories East Cluster Clinical Research Ethics Committee [2016.228].

Open Access This article is distributed under the terms of the Creative Commons Attribution 4.0 International License (http:// creativecommons.org/licenses/by/4.0/), which permits unrestricted use, distribution, and reproduction in any medium, provided you give appropriate credit to the original author(s) and the source, provide a link to the Creative Commons license, and indicate if changes were made.

\section{References}

1. Bevan ER, Jones AM, Hawkey PM. Global epidemiology of CTXM B-lactamases: temporal and geographical shifts in genotype. J Antimcirob Chemother 2017;72(8):2145-2155. https://doi.org/10. 1093/jac/dkx146

2. Chang YT, Coombs G, Ling T, Balaji V, Rodrigues C, Mikamo H et al (2017) Epidemiology and trends in the antibiotic susceptibilities of gram-negative bacilli isolated from patients with intraabdominal infections in the Asia-Pacific region, 2010-2013. Int J Antimicrob Agents 49:734-739. https://doi.org/10.1016/j. ijantimicag.2017.01.030

3. You JHS, Mok SSM, Chang CCM, Lee N, Ip M (2009) Bacteraemia with extended-spectrum $\beta$-lactamases-producing Escherichia coli in Hong Kong. Int J Antimicrob Agents 34:611612. https://doi.org/10.1016/j.ijantimicag.2009.07.015

4. Pau CKY, Ma FFT, Ip M, You JHS (2015) Characteristics and outcomes of Klebsiella pneumoniae bacteraemia in Hong Kong. Infect Dis (Auckl) 47:283-288. https://doi.org/10.3109/00365548. 2014.985710

5. Balakrishnan I, Awad-El-Kariem FM, Aali A, Kumari P, Mulla R, Tan B et al (2011) Temocillin use in England: clinical and microbiological efficacies in infections caused by extended-spectrum and/or derepressed AmpC $\beta$-lactamase-producing Enterobacteriaceae. J Antimicrob Chemother 66:2628-2631. https://doi.org/10.1093/jac/dkr317

6. Habayeb H, Sajin B, Patel K, Grundy C, Al-Dujaili A, Van de Velde S (2015) Amoxicillin plus temocillin as an alternative empiric therapy for the treatment of severe hospital-acquired pneumonia: results from a retrospective audit. Eur J Clin Microbiol Infect Dis 34:16931699. https://doi.org/10.1007/s10096-015-2406-x

7. El Sakka N, Gould IM (2016) Role of old antimicrobial agents in the management of urinary tract infection. Expert Rev Clin 
Pharmacol 9:1047-1056. https://doi.org/10.1080/17512433.2016. 1189325

8. Andrews JM, Jevons G, Walker R, Ashby J, Fraise AP (2007) Temocillin susceptibility by BSAC methodology. J Antimicrob Chemother 60:185-187. https://doi.org/10.1093/jac/dkm179

9. CLSI (2015) Methods for dilution antimicrobial susceptibility tests for bacteria that grow aerobically; approved standard, 10th edn. Clinical and Laboratory Standards Institute, Wayne, PA

10. CLSI (2015) Performance standards for antimicrobial susceptibility testing; twenty-fifth informational supplement. Clinical and Laboratory Standards Institute, Wayne, PA

11. Andrews JM. BSAC Susceptibility Testing Guide. Chapter 2. Determination of minimum inhibitory concentrations. http://www. bsac.org.uk/stewardship-surveillance/susceptibility/susceptibilitytesting-guide. Accessed 5 August 2017

12. Reynolds R, Shackcloth J, Felmingham D, MacGowan A (2003) Comparison of BSAC agar dilution and NCCLS broth microdilution MIC methods for in vitro susceptibility testing of Streptococcus pneumoniae, Haemophilus influenzae and Moraxella catarrhalis: the BSAC respiratory resistance surveillance programme. J Antimicrob Chemother 52:925-930

13. Seo MR, Kim SJ, Kim Y, Kim J, Choi TY, Kang JO et al (2014) Susceptibility of Escherichia coli from community-acquired urinary tract infection to fosfomycin, nitrofurantoin, and temocillin in Korea. J Korean Med Sci 29:1178-1181. https://doi.org/10. 3346/jkms.2014.29.8.1178

14. Kim B, Kim J, Seo MR, Wie SH, Cho YK, Lim SK et al (2013) Clinical characteristics of community-acquired acute pyelonephritis caused by ESBL-producing pathogens in South Korea. Infection 41:603-612. https://doi.org/10.1007/s15010-013-0441-z

15. Chen YT, Murad KA, Ng LSY, Seah JTH, Park JJ, Tan TY et al (2016) In vitro efficacy of six alternative antibiotics against multidrug resistant Escherichia coli and Klebsiella pneumoniae from urinary tract infections. Ann Acad Med Singapore 45:245-250 